\title{
Effects on Texture and Microstructure of Aluminium Alloy 7075-T651 during High Strain Rate Torsion
}

\author{
ShaileshTiwari $^{\mathrm{a}}$, and Manoj Chopkar ${ }^{\mathrm{a}}$ \\ ${ }^{a}$ Department of Metallurgical Engineering, National Institute of Technology, Raipur, India. \\ ${ }^{\mathrm{b}}$ Department of Materials Science and Engineering, Indian Institute of Technology, Kanpur, India. \\ Corresponding Author Email-id: mchopkar.met@nitrr.ac.in
}

\begin{abstract}
Deformation behaviour of aluminium alloy AA-7075 T651 was investigated at high strain rate using Torsional Split Hopkinson Bar (TSHB). Hexagonal grip cylindrical AA 7075 T651specimens were deformed at different angles of twist, giving rise to strain rate ranging from730s-1to $2041 \mathrm{~s}-1$. Comprehensive microstructural and textural investigation was carried out using electron back scatter diffraction (EBSD), X-ray line profile analysis and bulk texture measurement using X-rays for all the deformed samples. The high strain rate torsion tested samples showed a characteristic torsion texture for face centre cubic materials with a dominant " $A$ " $\left\{11 \overline{1}^{-}\right\}<110>\mid\{1 \overline{1} 1\}<1 \overline{1} \overline{0}>$ component and the overall texture weakened with increase in strain rate and strain. The T6 condition of alloy makes the matrix devoid of solute elements and thus reduces the stacking fault energy of the aluminium solid solution matrix and aids cross slip leading to a dominant "A" component.Microstructural parameters determined from X-ray diffraction using modified Williamson-Hall and Variance method further corroborated these results indicating the onset of dynamic recovery at the highest strain rate.
\end{abstract}

Keywords: Dynamic recovery, Microstructure, Strain rate, Texture, Torsion.

\section{Introduction}

AA 7075 has been the standard workhorse alloy in the aerospace industries since its inception in the year 1943 by Japanese company Sumitomo metal, [1]. An enviable combination of high mechanical strength, good fatigue resistance and adequate machinability makes this $\mathrm{Al}-\mathrm{Zn}-\mathrm{Mg}-\mathrm{Cu}$ alloy a suitable option for aerospace applications like aircraft fittings, gears and shafts and other structural components[2]. The high strength to weight ratio of $\mathrm{Al}-\mathrm{Zn}-\mathrm{Mg}-\mathrm{Cu}$ alloys can be attributed to interaction between mobile dislocations and finely dispersed precipitates [3]. However, these aircraft components are susceptible to dynamic impact loading in service, in which case strain rate of the order of $\sim 10^{3} \mathrm{~s}^{-1}$ can be achieved[4]. Such high strain rates can be achieved in the event of collision with flying debris or birds[4]. Consequently, catastrophic failure can occur or can cause severe maintenance and repair costs. Thus, it is of paramount importance to understand the deformation behaviour of $\mathrm{Al}-\mathrm{Zn}-\mathrm{Mg}-\mathrm{Cu}$ alloys under dynamic loading conditions. There is abundant literature available for high strain rate deformation under compression loading, while the same under torsion is scarce. Therefore, in the present investigation, split Hopkinson torsional bar has been used to decipher deformation behaviour of $\mathrm{Al}-\mathrm{Zn}-\mathrm{Mg}-\mathrm{Cu}$ alloys at a variety of strain rates ranging from $700 \mathrm{sec}^{-1}$ to $2000 \mathrm{sec}^{-1}$. The deformed microstructures were characterized using bulk texture measurements, X-ray line profile analysis and electron back scatter diffraction to understand and appreciate the operative micro-mechanisms of deformation.

\section{Experimental Details}

In the present investigation, extruded bars of diameter $30 \mathrm{~mm}$ of AA 7075 aluminium alloy in T651 temper were used with following compositions.

Table I: Chemical composition of AA 7075.

\begin{tabular}{llllllllll}
\hline & $\mathrm{Si}$ & $\mathrm{Fe}$ & $\mathrm{Cu}$ & $\mathrm{Mn}$ & $\mathrm{Mg}$ & $\mathrm{Cr}$ & $\mathrm{Zn}$ & $\mathrm{Ti}$ & $\mathrm{Al}$ \\
Zlements & & & & & & & & \\
\hline Wt \% & $\mathbf{1 . 1 2}$ & $\mathbf{1 . 2 5}$ & $\mathbf{1 . 6}$ & $\mathbf{1 . 0 6}$ & $\mathbf{2 . 6}$ & $\mathbf{0 . 2}$ & $\mathbf{5 . 8}$ & $\mathbf{1 . 0 7}$ & 3al. \\
\hline
\end{tabular}


The sample dimensions were as shown in Fig. 1.

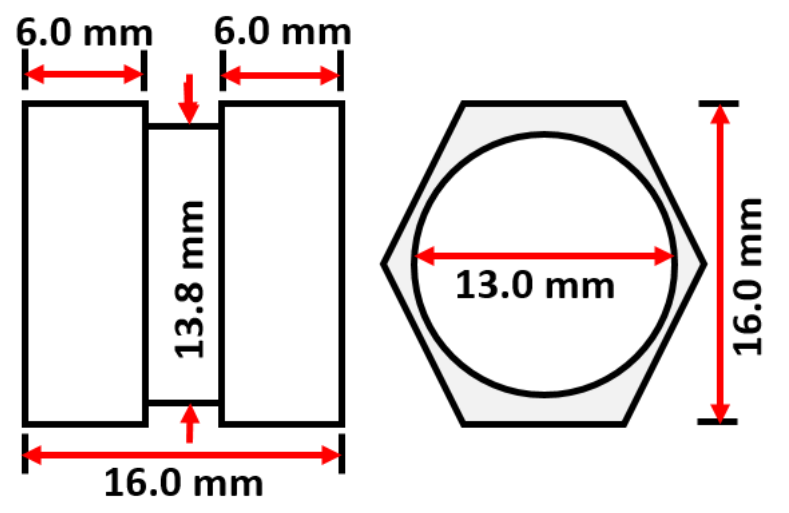

Fig. 1. Dimensions of the split Hopkinson torsional bar specimen.

The split Hopkinson torsional bar consists of two elastic bars, a clamp with a sudden releasing mechanism, and a loading arrangement to twist one of the bars[6]. The hollow cylindrical sample is placed between the bars and twist is applied at one end of the bar via a loading mechanism [6]. This results in the storage of torque between loading end of the bar and clamp. Subsequently, the sudden release of clamp results in propagation of shear stress pulse along the bar, thereby loading the sample in shear at high strain rate[6]. The different angles of twist used in the present investigation are 4, 10 and 16 degrees. The elastic wave data is acquired by oscilloscope connected to the strain gages mounted on both incident and transmitted bars. The elastic wave data is used to obtain stress strain and strain rate values for each test.

Microstructural analysis was carried out using a JEOL field emission gun scanning electron microscope (FESEM) equipped with electron back scatter diffraction (EBSD) set up from Oxford instruments. Transmission electron microscopy (TEM) was carried out using Tecnai $\mathrm{G}^{2}$ microscope operating at $200 \mathrm{kV}$. The samples for TEM were prepared via twin-jet electropolishing. X-ray diffractograms for line profile analysis were captured using Rigaku Miniflex 600. Si powder was used as standard to separate the broadening contribution from instrument.

\section{Data analysis}

The elastic wave data captured by the stain gages mounted on the incident and transmitted bar were used to determine the average strain rate $\left(\tilde{\gamma}_{s}\right)$.using equation $1[7]$.

$$
\gamma_{s}(t)=\frac{C_{s} D_{s}}{L_{s} D} *\left[\gamma_{I}(t)-\gamma_{R}(t)-\gamma_{T}(t)\right]
$$

where, $L_{g}$ and $D_{s}$ are mean gage length and diameter of the sample, $D$ is the diameter of the bar (25.4 mm), $C_{g}$ is the shear wave velocity in the bar $(3040 \mathrm{~m} / \mathrm{sec})$ and $\gamma_{I}(t), \gamma_{R}(t)$ and $\gamma_{T}(t)$ are the incident, reflected and transmitted strains, respectively. Eq. 1 can be integrated to obtain strain in the sample as a function of time.

The shear stress $\left(\tau_{\varepsilon}\right)$ in the sample can be calculated from the stress waves present in the incident and transmitted bars

$$
\tau_{s}(t)=\frac{G D^{\mathrm{a}}}{16 D_{s}^{2}} *\left[\gamma_{I}(t)+\gamma_{R}(t)+\gamma_{T}(t)\right]
$$

Here, $G$ is shear modulus (27 GPa) and all other terms are as defined previously. Thus, data obtained from equation 1 and 2 can be used to plot strain rate and stress as a function of strain.

\section{X-ray line profile analysis}

\subsection{Modified Williamson-Hall method}

The classical Williamson-Hall (W-H) plot can be used to separate different contributions to X-ray peak broadening[8]. 
Taking into account strain anisotropy a dislocation model was proposed by Ungar et al.[9]. The model was based upon anisotropic contrast of dislocations in diffraction. Therefore, the classical W-H equation was modified as

$$
\beta_{d} \cos \theta=\frac{0.9 \lambda}{t}+4 \varepsilon \sqrt{C_{\text {avg }}} \sin \theta
$$

Here, $C_{\text {avg }}$ is the average dislocation contrast factor for each diffraction peak.

Table II: Dislocation contrast factor for edge and screw dislocations.

\begin{tabular}{ccccc}
\hline $\begin{array}{c}\text { Dislocation contrast } \\
\text { factor }\end{array}$ & 111 & 200 & 220 & 311 \\
\hline Edge dislocations & $\mathbf{0 . 1 8}$ & $\mathbf{0 . 1 9}$ & $\mathbf{0 . 1 8}$ & $\mathbf{0 . 1 9}$ \\
\hline Screw dislocations & $\mathbf{0 . 0 6}$ & $\mathbf{0 . 2 3}$ & $\mathbf{0 . 1 0}$ & $\mathbf{0 . 1 5}$ \\
\hline
\end{tabular}

\subsection{Variance method}

The microstructural parameters can be obtained by another indirect technique called variance range method[10]. The variance $(W)$ of a region $\pm \sigma$ can be expressed as

$$
W(\sigma)=\frac{\int_{2 \theta_{0}-\sigma}^{2 \theta_{0}+\sigma}\left(2 \theta-2 \theta_{0}\right)^{2} I(2 \theta) d(2 \theta)}{\int_{-\infty=\infty}^{+\infty} I(2 \theta) d(2 \theta)}
$$

where, $2 \theta_{0}$ is the centroid position and $I$ is the intensity.

\section{Results and Discussion}

\subsection{Starting material}

The (111) and (200) pole figures and crystal orientation map of AA 7075 is shown in Fig. 2. The texture of the AA 7075 is characterized by thepresence of strong $\{111\}<11 \overline{2}>\mathrm{P}\left(\mathrm{B}_{\mathrm{ND}}\right)$ and weak $\{001\}<100>$ Cube component. The microstructure consists ofelongated grains, typically found in hot extruded alloys.

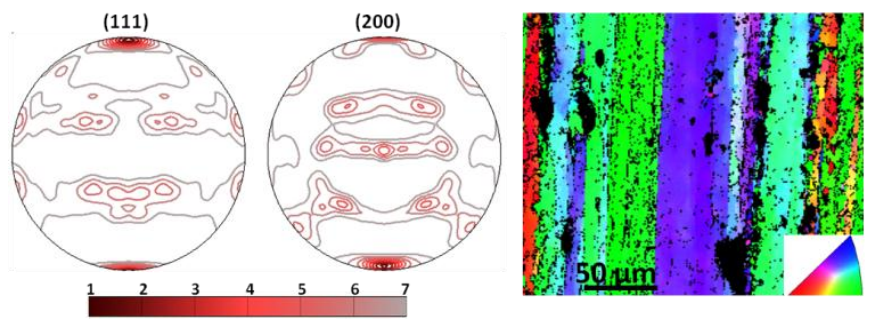

Fig. 2. (111) and (200) pole figures and crystal orientation map of the starting sample

\subsection{Mechanical properties}

Fig. 3 shows the typical dynamic strain rate versus strain and stress-strain curves for samples deformed at different angles of twist. Maximum strain rate achieved as a function of angle of twist is summarized in Table III. Maximum strain rate of $2041 \mathrm{sec}^{-1}$ is achieved for the sample twisted by sixteen degrees, while minimum strain rate of $730 \mathrm{sec}^{-1}$ is achieved for the sample twisted by four degrees. The maximum flow stress increased as the angle of twist was increased from $4^{\circ}$ to $16^{\circ}$ (Table III). In a similar fashion, the higher angle of twist the higher the total amount of energy absorbed during plastic deformation.The energy absorbed was calculated based on. area under the stress-strain curves. 

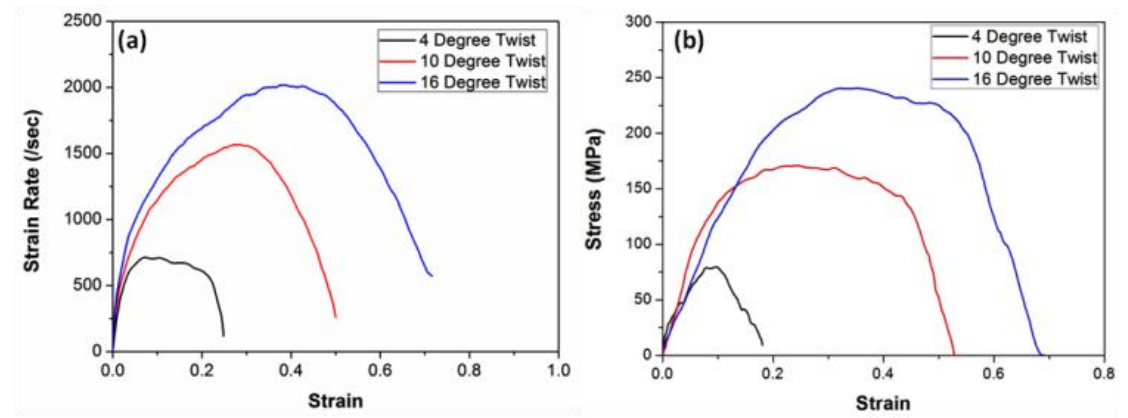

Fig. 3. (a) Strain rate versus strain and (b) Stress versus Strain for high strain rate torsional Split Hopkinson bar

Table III: Mechanical properties as a function of angle of twist.

\begin{tabular}{lccc}
\hline $\begin{array}{c}\text { Angle of } \\
\text { twist }\end{array}$ & Strain rate $\left(\mathrm{sec}^{-1}\right)$ & Shear stress $(\mathrm{MPa})$ & Total energy absorbed (KJ) \\
\hline $4 \mathrm{deg}$ & $\mathbf{7 3 0}$ & $\mathbf{8 0}$ & $\mathbf{1 0}$ \\
\hline $10 \mathrm{deg}$ & $\mathbf{1 5 8 5}$ & $\mathbf{1 7 1}$ & $\mathbf{7 0}$ \\
\hline $16 \mathrm{deg}$ & $\mathbf{2 0 4 1}$ & $\mathbf{2 4 1}$ & $\mathbf{1 1 6}$ \\
\hline
\end{tabular}

The microstructure in T651 temper can be characterized by the presence of finely dispersed coherent $\eta^{\prime}$ precipitates (Fig. 4).

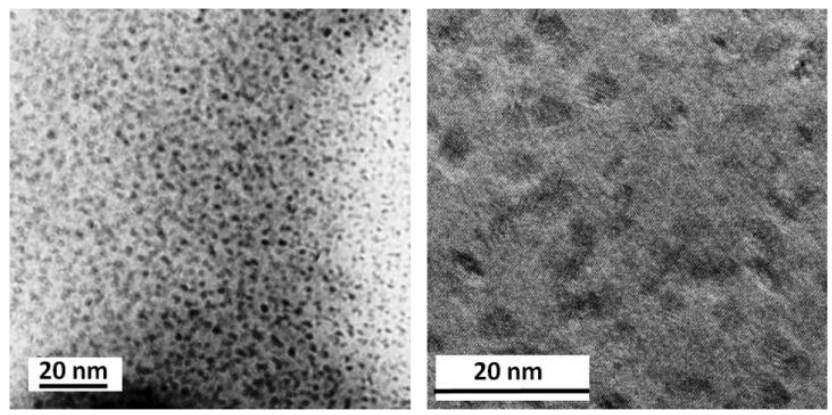

Fig. 4. $\eta$ ' precipitates in AA 7075 alloy

\subsection{Evolution of crystallographic texture}

Fig. 5 shows the (111) and (200) pole figures for AA 7075 samples deformed at different angles of twist. AA 7075 samples deformed at low $\left(4^{\circ}\right)$ as well as high $\left(16^{\circ}\right)$ strain rate, showed a typical shear texture observe in FCCmaterials. Shear texture in FCC materials is characterized by the presence of various components like A $\{1 \overline{1}\}<110>/\{111\}<\overline{11} 0>, \mathrm{B}\left\{[112\}<110>/\{1 \overline{1} \overline{2}\}<\overline{11} 0>, \mathrm{C}\{001\}<110>, \mathrm{A}_{1}{ }^{*}\{\overline{1} 1\}<112>\right.$ and $\mathrm{A}_{2}{ }^{*}\{1 \overline{1} 1\}<112>[12]$. The $\mathrm{A}, \mathrm{A}_{1}{ }^{*}, \mathrm{~A}_{2}{ }^{*}$ components are located along the $\{111\}<u v w>$ fibre while the $\mathrm{B}$ and $\mathrm{C}$ components are located along the $\{h k l\}<101>$ fibre in the Euler space[12]. As evident from the pole figures (Fig. 5), A $\{1 \overline{1} 1\}<110>/[111\}<\overline{11} 0>$ component dominates over other texture components for the samples deformed at different strain rates. In general for FCC materials, texture is dominated by A, B and A* component at lower strains, while at higher strains, texture is dominated by $\mathrm{C}$ component[13]. Since the strain achieved in the present investigation is around $\sim 0.3-0.5$, A $\{1 \overline{11}\}<110>/\{111\}<\overline{11} 0>$ is expected to be the dominant texture component.

Since the alloy is in T6 condition, the matrix is devoid of solute elements which reduce the stacking fault energy of the aluminium solid solution matrix and aid in cross slip leading to a dominant A component.

A slight decrease in strength of texture is observed at high strain rates even with increase in strain. A higher value of strain rate sensitivity was used by Canova et al.[5] to model texture evolution during high strain rate deformation. According to their model, texture weakening should occur for high strain rate compression or tension, while strengthening of texture should occur for high strain rate shear deformation. The increase in number of active slip systems was considered to be the major reason behind weakening of texture during high strain rate compression, while instability of orientations was the main reason for strengthening of texture during shear deformation. However, due to unavailability of sufficient time during high strain rate deformation, the entire grain cannot reorient itself, thereby causing grain fragmentation and weakening of texture. Therefore, it 
can be concluded that grain fragmentation during high strain rate deformation is the main reason behind weakening of texture.

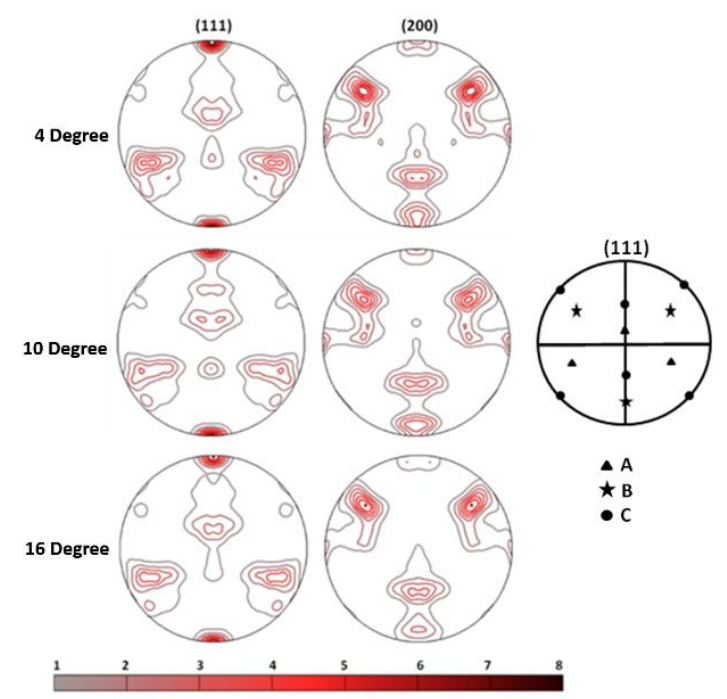

Fig. 5. (111) and (200) pole figures of sample twisted at different degrees

\subsection{Evolution of microstructure}

Band contrast and crystal orientation maps of the differently processed samples are shown in Fig. 6 (a-c). The microstructure mainly consists of grains elongated along extrusion direction with few equiaxed grains of size $\sim 5 \pm 6 \mu \mathrm{m}$. The unindexed points correspond to smearing of Kikuchi patterns of various pixels and which could not be indexed by the orientation imaging microscopy (OIM) system. High strain concentration inside these pixels renders the pixel area unidentifiable with a solitary zone axis. The heterogeneity of microstructure is clearly evident in terms of color gradients inside the grains, indicating development of orientation gradients during plastic deformation(Fig. 6).

Evolution of dislocation density, misorientation angle and subgrain formation is expected to occur during high strain rate deformation.Intragranular misorientation parameters like local average misorientation (LAM) and grain orientation spread (GOS) were used for further characterization of the microstructure.
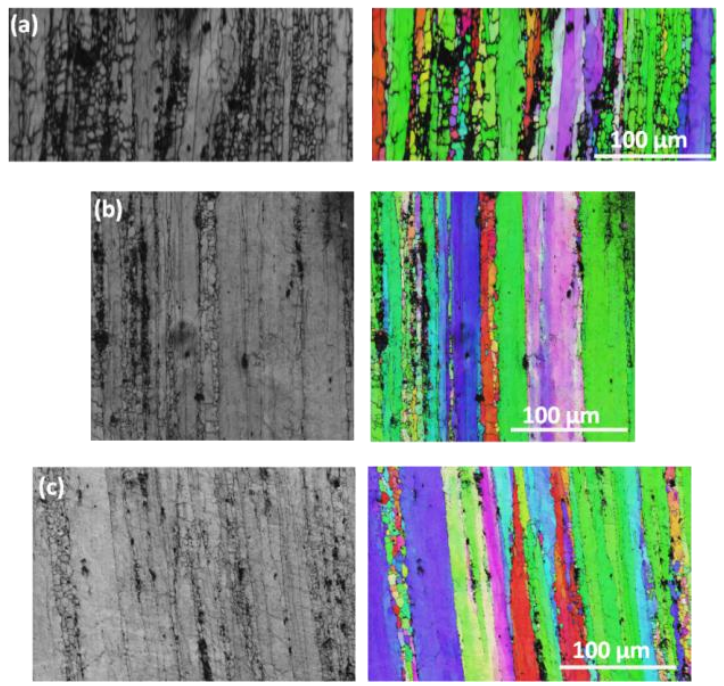

Fig. 6. Band contrast and crystal orientation maps of differently processed samples (a) $4^{\circ}$ (b) $10^{\circ}$ (c) $16^{\circ}$.

LAM parameter is very effective in capturing small orientation changes on the map and thus, highlights regions of higher deformation. The average misorientation between a pixel and its surrounding pixels is calculated and a mean value is assigned to that pixel[14]. LAM parameter isvery sensitive to step size. Thus, it was ensured that the step size remains the same for each EBSD scan. 
Intragranular misorientation parameters suggest that restoration processes like dynamic recovery are in operation for the $16^{\circ}$ twisted sample. The basic mechanisms of dynamic recovery is dislocation cross slip and glide which results in formation of subgrains and low angle grain boundaries[15]. As shown in Fig. 7, the fraction of low angle grain boundaries is highest for the $16^{\circ}$ twisted sample. At high strains, the microstructure is generally refined by conversion of low angle grain boundaries (LAGB) to high angle grain boundaries (HAGB). However, for high strain rate deformation, conversion from LAGB to HAGB is retarded due to less time available for dislocation rearrangement and interaction[16]. In addition to dynamic recovery, this can also be considered as another reason for higher amount of LAGB in $16^{\circ}$ twisted sample.

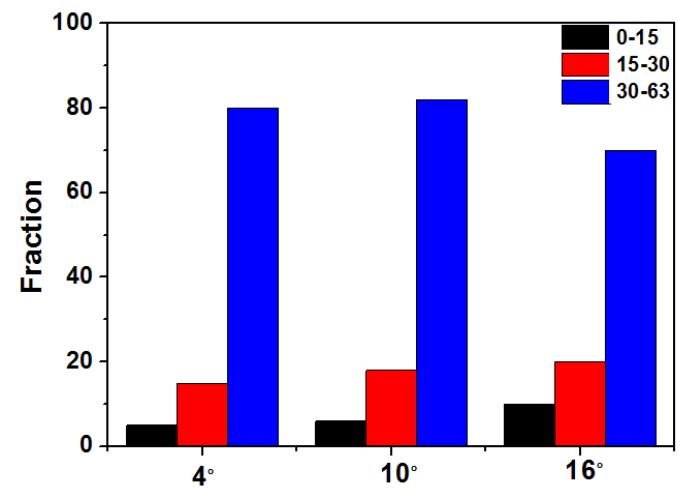

Fig. 7. Grain boundary character distribution for differently processed samples

The combined operation of strain hardening and dynamic recovery causes continuous formation and annihilation of dislocations, thereby ensuring constant dislocation density within the dislocation substructure. After a strain of $\sim 0.4-0.5$, the dislocation substructure reaches a steady state with approximately constant mean size and misorientation[15].

\subsection{X-ray line profile analysis}

The X-ray diffractograms of the samples deformed at different angles of twist are shown in Fig. 8. Generally, with increasing deformation there is shifting and broadening of X-ray peaks. As can be seen from Fig. 8, Full Width Half Maximum (FWHM) value increases with angle of twist from $4^{\circ}$ to $10^{\circ}$, indicating refinement in crystallite size and presence of micro-strain. However, FWHM value changes marginally with further increase in angle of twist to $16^{\circ}$.

Modified W-H plots for the samples deformed at different strain rates are shown in Fig. 9. For each sample, a least square fitting line is drawn with corresponding coefficient of regression $\left(\mathrm{R}^{2}\right)$. As evident from the plots, best linear fit for $4^{\circ}$ sample is obtained assuming $90 \%$ screw and $10 \%$ edge dislocations. With increase in angle of twist from $4^{\circ}$ to $10^{\circ}$, best linear fit is now obtained assuming $50 \%$ edge and $50 \%$ screw dislocations. Following a similar pattern, for $16^{\circ}$ twisted sample, best fit is obtained assuming $80 \%$ edge and $20 \%$ screw dislocations. Therefore, it can be concluded that with increase in angle of twist or strain rate, percentage of screw dislocations in the material is decreasing.
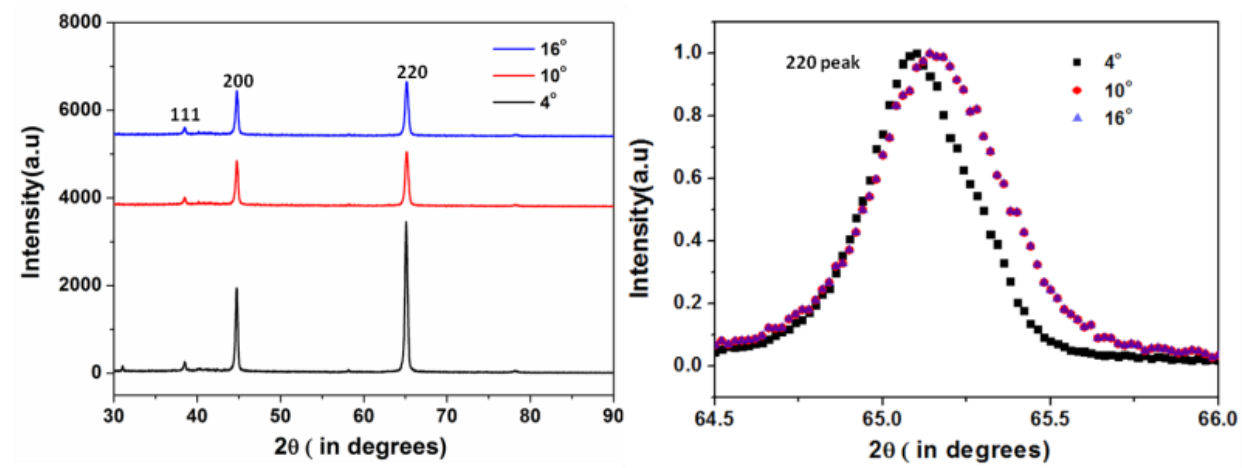

Fig. 8.X ray diffractograms of the samples deformed at different angles of twist 
Microstructural parameters obtained using variance method are summarized in Table IV. With increase in angle of twist from $4^{\circ}$ to $10^{\circ}$, crystallite size decreases and micro-strain increases, thereby causing the dislocation density to increase. However, there is no significant change in crystallite size, micro-strain and dislocation density with further increase in angle of twist to $16^{\circ}$. Occurrence of dynamic recovery at such high strain rates can provide a suitable explanation of the observed behavior. In materials of high stacking fault energy like Al, constrictions in stacking fault ribbon can occur readily, thereby permitting dislocation cross slip[13]. In addition, temperature rise associated with high strain rate deformation also provides the energy required for recombination of partial dislocations. Therefore, dynamic recovery is rapid and extensive at such high strain rates [11]. With increase in angle of twist from $4^{\circ}$ to $10^{\circ}$, dislocation density increases as dislocations tend to interact and multiply. However, as the dislocation density increases, driving force for recovery also increases[15]. After a certain strain, a steady state equilibrium condition is attained wherein the rates of dislocation accumulation and annihilation are equal[15]. In such a scenario, dislocation density remains constant and a steady state flow stress is obtained. Therefore, present results suggest that with increase in angle of twist from $10^{\circ}$ to $16^{\circ}$, a steady state equilibrium sets in causing dislocation density to be similar for both the samples.

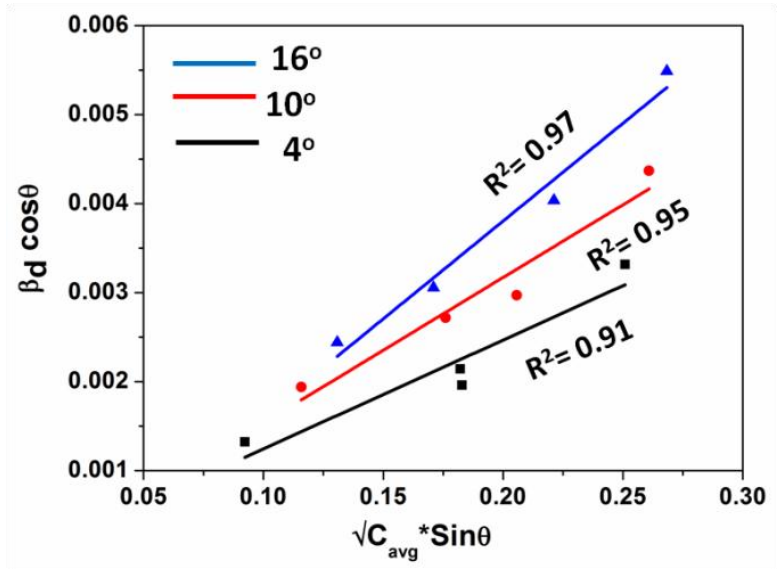

Fig. 9. Modified Williamson-Hall analysis of samples deformed at different angles of twist.

\begin{tabular}{cccc} 
Table IV: Microstructural parameters obtained using Variance method. \\
\cline { 2 - 4 } & $\begin{array}{c}\text { Crystallite Sizt } \\
(\mathrm{nm})\end{array}$ & $\begin{array}{c}\text { R.M.S. } \\
\text { Strain }\end{array}$ & $\begin{array}{c}\text { Dislocation } \\
\text { Density }\left(\mathrm{m}^{-2}\right)\end{array}$ \\
\hline $\begin{array}{c}\mathbf{1 . 7 2 E - 0 8} \\
\text { Degrees }\end{array}$ & $\mathbf{0 . 0 0 1 1 9 4}$ & $\mathbf{1 . 0 2 7 9 2 E + 1 5}$ \\
\hline $\begin{array}{c}10 \\
\text { Degrees }\end{array}$ & $\mathbf{6 . 4 7 E - 0 9}$ & $\mathbf{0 . 0 0 3 1 9 6}$ & $\mathbf{7 . 3 2 6 5 8 E + 1 5}$ \\
\hline 16 & $\mathbf{6 . 7 E - 0 9}$ & $\mathbf{0 . 0 0 3 1 4}$ & $\mathbf{6 . 9 5 6 5 9 E + 1 5}$ \\
Degrees & & & \\
\hline
\end{tabular}

\section{Conclusions}

Textural and microstructural evolution during high strain rate torsion of AA 7075 in T651 temper was studied using a torsional Split Hopkinson bar. The samples were subjected to deformation at different angles of twist $\left(4^{\circ}\right.$, $10^{\circ}$ and $16^{\circ}$ ) leading to different strain rate and strain. Following are the major findings of the present work:

(i) The maximum shear stress was observed for specimens deformed using the highest angle of twist of $16^{\circ}$. The stress-strain curves exhibited negligible work hardening at all the strain rate samples.

(ii) A slight weakening of texture was observed in specimens loaded using the angle of twist of $16^{\circ}$ twisted sample (highest strain rate and strain). All the samples showed ' $A$ ' component to be the dominant texture component.

(iii) EBSD analysis of samples deformed at different angles of twist indicated that intragranular misorientation parameters like LAM and GOS are lower for specimens loaded using $16^{\circ}$ angle of twist compared to that subjected to $10^{\circ}$ angle of twist. The $16^{\circ}$ twisted sample showed higher fraction of low angle grain boundaries confirming occurrence of dynamic recovery. 
(iv) X-ray line profile analysis revealed that microstructural parameters like crystallite size, micro-strain and dislocation density are similar for $16^{\circ}$ and $10^{\circ}$ twisted sample, further confirming the existence of dynamic recovery at such high strain rates.

\section{Acknowledgements}

The authors express their acknowledgements to Prof. Satyam Suwas, IISc, Bangalore and Dr. Nilesh Gurao, IIT Kanpur for their valuable inputs. Experimental facility provided by Dr. J. Szpunar and Dr. A. Odeshi, Department of Mechanical Engineering, University of Saskatchewan, Saskatoon, Canada. The authors are grateful to Texture Laboratory at the Advanced Centre for Materials Science, Indian Institute of Technology Kanpur for providing the characterization facilities used in the present investigation. Thanks are due to NRC-M at IISc, Bangalore. The authors would like to thank Science and Engineering Research Board, India for funding the present work under project no: SB/FTP/ETA-122/2013.

\section{References}

[1] W. Shu, L. Hou, C. Zhang, F. Zhang, J. Liu, J. Liu, L. Zhuang, J. Zhang, Materials Science and Engineering: A, 657 (2016) 269-283.

[2] H. Fang, H. Chao, K. Chen, Materials Science and Engineering: A, 610 (2014) 10-16.

[3] D. Liu, B. Xiong, F. Bian, Z. Li, X. Li, Y. Zhang, Q. Wang, G. Xie, F. Wang, H. Liu, Materials Science and Engineering: A, 639 (2015) 245-251.

[4] A. Ghosh, A. Adesola, J. Szpunar, A. Odeshi, N. Gurao, Materials \& Design, 81 (2015) 1-10.

[5] G.R. Canova, C. Fressengeas, A. Molinari, U.F. Kocks, Acta Metall., 36 (1988) 1961-1970.

[6] J. Duffy, J. Campbell, R. Hawley, J. Appl. Mech., 38 (1971) 83-91.

[7] J. Lifshitz, H. Leber, Int. J. Impact Eng., 15 (1994) 723-733.

[8] A.K. Zak, W.A. Majid, M.E. Abrishami, R. Yousefi, Solid State Sci., 13 (2011) 251-256.

[9] T. Ungár, A. Borbély, Appl. Phys. Lett., 69 (1996) 3173-3175.

[10] F. Sánchez-Bajo, F. Cumbrera, J. Appl. Crystallogr., 30 (1997) 427-430.

[11] S. Nemat-Nasser, Y.-F. Li, J.B. Isaacs, Mech. Mater., 17 (1994) 111-134.

[12] L.S. Tóth, K.W. Neale, J.J. Jonas, Acta Metall., 37 (1989) 2197-2210.

[13] F. Montheillet, M. Cohen, J.J. Jonas, Acta Metall., 32 (1984) 2077-2089.

[14] S.I. Wright, M.M. Nowell, D.P. Field, Microsc. Microanal., 17 (2011) 316-329.

[15] A. Rollett, F. Humphreys, G.S. Rohrer, M. Hatherly, Recrystallization and related annealing phenomena, Elsevier, 2004.

[16] N. Nayan, N.P. Gurao, S.V.S. Narayana Murty, A.K. Jha, B. Pant, S.C. Sharma, K.M. George, Materials \& Design (1980-2015), 65 (2015) 862-868

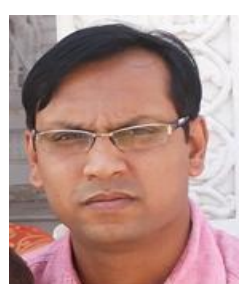

"Authors Biodata"

Manoj Kumar Chopkar is Assistant professor in Department of Metalurgical Engineering, National Institute of Technology, Raipur, India. He obtained his Ph.D. from Indian Institute of Technology, Kharagpur. His current field of interest are synthesis and characterisation of Nanomaterial and Nanofluid, Heat Transfers in Nanofluid, Nanostructured Steel, Nanostructured and Amorphous materials for structural application, Phase Transformation (solid state) and High Strain rate deformation.

Shailesh Tiwari is a doctoral student in the field of 'high strain rate deformation and related texture studies' in the department of Metallugical Engineering at the National Institute of Technology, Raipur. He has done his Master of Technology from Indian Institute of Technology, Kanpur 